\title{
Factores limitantes en el acceso y utilización del componente de crecimiento y desarrollo en niños de 0 a 5 años, ESSALUD, Huancayo, 2014
} Limiting factors in the access and use of the growth and development component in children from 0-5 years old, ESSALUD, Huancayo, 2014

\author{
César Augusto Reyes Luján' \\ Universidad Nacional del Centro del Perú \\ cesarlujan77@hotmail.com
}

\author{
Iris Yone Carrasco Díaz ${ }^{2}$ \\ Universidad Nacional del Centro del Perú \\ icarrasco77@hotmail.com
}

\section{RESUMEN}

Se estableció como objetivo determinar los factores limitantes y su asociación con el acceso y utilización del componente de crecimiento y desarrollo en niños de 0 a 5 años en el Centro de Atención Primaria II, Chilca, ESSALUD 2014, en la provincia de Huancayo. Investigación de alcance correlacional, diseño transversal y retrospectivo. La población fue de 3806 niños menores de 5 años asegurados, la muestra fue de 350 niños. Se utilizaron estadísticos descriptivos, distribución de frecuencias, tablas de contingencia y Chi cuadrado. Se tiene como resultados que el $10 \%$ de los niños no utilizan muy frecuentemente el componente; 12,5 \% de nivel socioeconómico medio asisten poco frecuente en relación al nivel socioeconómico alto que es 2,3\%; $77,3 \%$ de las madres que viven lejos asisten muy frecuentemente a diferencia de los que viven cerca que es del 93,2\%; $22 \%$ de los que asisten menos frecuentemente consideran que no es suficiente el número de profesionales a diferencia del $7 \%$ que dicen que si es suficiente. Algunas conclusiones indican que los factores limitantes asociados con el acceso y utilización del componente de Crecimiento y Desarrollo son: La inadecuada e insuficiente promoción del componente, la percepción de la calidad de atención, características socioeconómicas del usuario, creencias sobre la salud del niño, desconocimiento del usuario del significado real del componente, las características de la organización interna del establecimiento y la accesibilidad geográfica.

Palabras claves: Crecimiento y desarrollo, factores limitantes, centro de atención primaria, componente.

\begin{abstract}
The goal of the study was to determine the limiting factors and their association with access and use of the growth and development component in children from 0-5 years old of the Primary Care Center II, Chilca, ESSALUD 2014 in the province of Huancayo. The research is of correlational scope, transversal and retrospective design. The population was represented by 3806 health-ensured children under the age of five, the sample was 350 children. Descriptive statistics, frequency distribution, contingency tables, and the Chi square test were used. As a result, $10 \%$ of children do not use the component frequently; $12,5 \%$ of middle class rarely attend the facility compared to $2,3 \%$ of the upper class; $77,3 \%$ of mothers living far away attend frequently, unlike the 93,2\% who live nearby, $22 \%$ of those who attend less frequently consider that the number of professionals is insufficient as opposed to the $7 \%$ who claims that it is enough. Some findings indicate that the limiting factors associated with the access and uses of the Growth and Development component are: inadequate and insufficient promotion component, the perception of quality of care, user socioeconomic characteristics, beliefs regarding the children's health, user's ignorance of the component's real meaning, the characteristics of the internal organization of the establishment and geographic accessibility.
\end{abstract}

Keywords: Growth and development, limiting factors, primary care center, component.

Historial del artículo:

Recibido: 15 de enero de 2014. Aprobado: 24 de marzo de 2015. Disponible en línea: 30 de junio de 2015

1 Médico Cirujano, Magister en Gestión de Servicios de salud, Director del CAP II Chilca ESSALUD, investigador de la Universidad Nacional del Centro del Perú.

2 Ing.Industrial, Dra. en Administración de la Educación, investigadora de la Universidad Nacional del Centro del Perú, docente de la

Universidad Continental. 


\section{INTRODUCCIÓN}

La atención primaria, el modelo de atención integral de Salud y la cartera de servicios en ESSALUD, son el eje fundamental de la preocupación por mejorar la cobertura y la accesibilidad de los pacientes asegurados en los establecimientos del primer nivel de atención. El CAP II Chilca es un establecimiento de primer nivel de atención de ESSALUD cuya jurisdicción comprende a 16 distritos, y donde la inclusión social es parte de la vivencia en la atención de los pacientes en especial a los menores de 5 años, sobre todo en el componente de Crecimiento desarrollo base fundamental de la atención en nuestro país teniendo en cuenta las altas tasas todavía de desnutrición crónica y anemia, por lo que es importante investigar en esta área de atención primaria para reconocer el impacto del trabajo diario de los establecimientos del primer nivel de atención del seguro social como un aporte a superar los indicadores de pobreza en este país.

El período en el que transcurren el crecimiento (el incremento en el peso y la talla de los niños) y el desarrollo (la maduración de las funciones del cerebro y de otros órganos vitales) se convierte en crítico, porque es durante ese lapso que el cerebro se forma y madura a la máxima velocidad. Siendo el cerebro el centro del desarrollo del niño las condiciones del ambiente intrauterino y del entorno familiar después del nacimiento determinan, en buena parte el potencial de capacidades que éste tendrá en su vida futura (1).

La nutrición juega un papel importante en el desarrollo del niño y el objetivo en el país es reducir la desnutrición infantil, reducir la prevalencia de desnutrición crónica y anemia en niñas y niños menores de 5 años, especialmente de las regiones con mayor pobreza siendo una de las principales estrategias el control de crecimiento y desarrollo (componente de CRED) (2).

La desnutrición crónica existente y la anemia son los principales problemas e indicadores de salud infantil a nivel mundial; la desnutrición es una de las causas subyacentes en aproximadamente la tercera parte de las defunciones infantiles, disminuyendo el nivel de desarrollo de un país. Lograr su disminución contribuirá garantizar el desarrollo de la capacidad física, intelectual, emocional y social de las niñas y niños.

En el mundo la proporción de niños menores de 5 años con insuficiencia ponderal (según los patrones de crecimiento infantil de la OMS) descendió del 25 \% en 1990 al $18 \%$ en 2005 (3). En algunos países, la prevalencia de la desnutrición ha aumentado y en 2005 todavía había en el mundo unos 186 millones de niños menores de 5 años con retraso del crecimiento, cuyas tres cuartas partes se registran en África y Asia. Según la UNICEF (2007), la desnutrición afecta a
53 millones (10\%) de personas en América Latina y el Caribe, de esta cifra casi 9 millones (16\%) son niñas y niños menores de cinco años que padecen desnutrición crónica o retardo en la talla. Asimismo, la anemia es el problema nutricional más grave y se extiende a lo largo de todos los países sin diferenciar estratos socio-económicos, mostrando prevalencias en niñas y niños menores de 5 años de alrededor del 50 $\%$; este promedio es superior en Haití $(65,8 \%)$ y en países de la Sub-región Andina como Bolivia (51,6\%), Perú $(50,4 \%)$ y Ecuador (50,2 \%) (4).

En el Perú de acuerdo con el Censo de Población y Vivienda del año 2009 la población infantil de 0 a 5 años llego a 3557113 habitantes. En el periodo 2009 - 2011 (ENDES) primer semestre, la desnutrición crónica a nivel nacional descendió en dos puntos porcentuales, al pasar de $23,8 \%$ a $21,6 \%$ afectando cerca de la cuarta parte de la población menor de 5 años; entonces la población de niños con deficiencia nutricional alcanza a más de 900 mil niños, el promedio nacional esconde las grandes diferencias entre la región Sierra $(37,1 \%)$ y Selva $(28,5 \%)$ en comparación con la región Costa $(15,1 \%)$ que tendrán un menor desarrollo físico y verán seriamente afectadas sus capacidades cognoscitivas y que estarán más afectas a enfermedades, lo que continuara afectando el capital humano de nuestro país en el futuro, puesto que este no es un problema nuevo. Los departamentos más afectados son: Huancavelica $(44,7 \%)$, Cajamarca $(32,0 \%)$, Huánuco $(31,0 \%)$, Apurímac (30,9\%) y Ayacucho (30,3 \%). Junín con un promedio de $54,4 \%$ de niños menores de 5 años con anemia se encuentra entre los 12 departamentos con mayor prevalencia en el país $(5,6)$.

Cifras preocupantes son la desnutrición crónica infantil en niños menores de 5 años y la anemia en niños entre 6 y 36 meses de edad, prácticamente no disminuyeron durante el año 2010. En ambos casos, según las cifras de la Encuesta Demográfica y de Salud Familiar - ENDES 2010, la disminución fue de menos de un punto porcentual, la cifra del $30,4 \%$, representa una disminución de 3,3 puntos porcentuales en relación al registrado en el 2009, que alcanzó 33,7 \%. En tanto que las cifras de anemia en la región oscila en un 41,4 $\%(6,7)$.

De acuerdo al Ministerio de Salud (MINSA) en el 2007, el control de crecimiento y desarrollo en los últimos años se observa un descenso en la captación de niños para esta actividad, a nivel nacional el porcentaje de niños controlados menores de 5 años es de 45,3\%, teniendo como niños controlados menores de un año al 69,22\%, niños de 1 año controlados $44,40 \%$ y niños controlados de 2 a 4 años de 37,49\%, disminuyendo el porcentaje según el niño va creciendo; en relación al porcentaje de niños que tiene riesgo en su desarrollo se evidenció que en los menores de un año el porcentaje 
fue de $5,27 \%$, de niños de 1 año 6,52 \% y niños de 2 a 4 años $3,28 \%$ (1).

En referencia al departamento de Junín (DIRESA) menciona que el año 2008 se han realizado a nivel regional 112813 atenciones de control a niños menores de 1 año, logrando solo 7973 niños controlados $(33,9 \%)$, a nivel de provincias tenemos a Satipo que ha realizado el mayor porcentaje de controlados $48,8 \%$, seguido de la provincia de Yauli con 43,9\%; la provincia con las más bajas coberturas de control de menores de 1 año son Junín $(24,3 \%)$, Chupaca $(26,8 \%)$, Huancayo $(29,1 \%)$, Jauja $(30,7 \%)$, concepción $(33,1 \%)(8)$.

En relación a lo expuesto anteriormente, el componente de crecimiento y desarrollo (CRED) según el MINSA (2009), es una de las actividades prioritarias que puede contribuir a enfrentar la problemática de salud infantil; dicho componente es el conjunto de actividades periódicas y sistemáticas que se realizan en la atención al niño de cero a nueve años, con el fin de detectar oportunamente cambios y riesgos en su estado de salud a través de un seguimiento; y es preocupante que siendo el programa de CRED uno de los principales ejes en la lucha contra la desnutrición infantil, solo accedan a este servicio la tercera parte de la población infantil (2).

La información estadística nos muestra lo que diversos estudios señalan y que es además una característica común a los países de América Latina, que la malnutrición infantil no depende principalmente de la falta de alimentos, sino que depende de factores: Mala salud materna, inadecuadas prácticas de alimentación, de cuidado infantil y falta de acceso a los servicios de salud infantil; entonces podremos mejorar el estado nutricional de la población infantil si mejoramos la educación, la atención y el acceso oportuno y de calidad a los servicios de salud y sanitarios a la población infantil (CRED); para evitar estos problemas de salud infantil es necesario poner mayor énfasis en la prevención y promoción en este grupo etario, esto a través de los programas de salud como es el Componente de Crecimiento y Desarrollo.

Lo que se busca con este trabajo de investigación es: a) Determinar los factores limitantes y su asociación con el acceso y utilización del componente de crecimiento y desarrollo mejorando las políticas de salud en relación a este grupo etario; b) Determinar los factores limitantes y su asociación con el acceso y utilización del componente de crecimiento y desarrollo; c) Asociar las variables socioeconómicas del usuario con el acceso y utilización del componente de crecimiento y desarrollo; d) Asociar el significado del componente CRED para la madre y la percepción del estado nutricional del niño con el acceso y utilización del componente de crecimiento y desarrollo; e) Asociar la variable de accesibilidad geográfica con el acceso y utilización del componente de crecimiento y desarrollo; f) Asociar las características de la organización interna del establecimiento de salud con el acceso y utilización del componente de crecimiento y desarrollo; g) Asociar la calidad de atención al usuario y la promoción del programa con el acceso y utilización del componente de crecimiento y desarrollo; y h) Medir la cobertura de crecimiento y desarrollo en niños de 0 a 5 años.

\section{MATERIAL Y MÉTODOS}

El nivel de investigación fue descriptivo, transversal y alcance correlacional (9). La población considerada fue de 3806 madres con hijos menores de 5 años de edad (10). La muestra fue de 349 madres con hijos menores de 5 años con un margen de error permitido de $5 \%$, un factor $P$ de 0,5 y $Q$ de 0,5 , que son los máximos valores para esta proporción. Se aplicó un muestreo aleatorio.

La técnica de recolección de datos fue la encuesta y el instrumento fue el cuestionario (9), con preguntas cerradas y abiertas ya que se trató de encontrar los factores limitantes en el acceso y utilización del componente de crecimiento y desarrollo y contiene 3 capítulos: En el capítulo I consignado como datos generales de la madre o responsable, datos generales del hijo y la variable dependiente (frecuencia de utilización); en el capítulo II y III incluyen las variables independientes; el capítulo II consignado como factores limitantes del lado de la demanda/usuario incluyendo en ella las variables socioeconómicas, y creencias en salud y el capítulo III consignado como factores limitantes del lado de la oferta/servicio que incluye aspectos de la accesibilidad geográfica, características de la organización interna, calidad de atención al usuario y promoción del componente de crecimiento y desarrollo (CRED).

El procesamiento de los datos se realizó utilizando software especializado, estadísticas como distribución de frecuencias y tablas de contingencias o tablas cruzadas. Para probar las hipótesis, por la naturaleza de las variables fueron realizadas pruebas de Chi cuadrado para determinar la relación entre las variables.

\section{RESULTADOS}

La figura $N^{\circ} 1$ muestra la frecuencia de utilizacion del componente de crecimiento y desarrollo es "muy frecuente" en un $90 \%$; "poco frecuente " en un $40 \%$; "casi frecuente" en un $5 \%$ y "muy poco frecuente" en $1 \%$. 


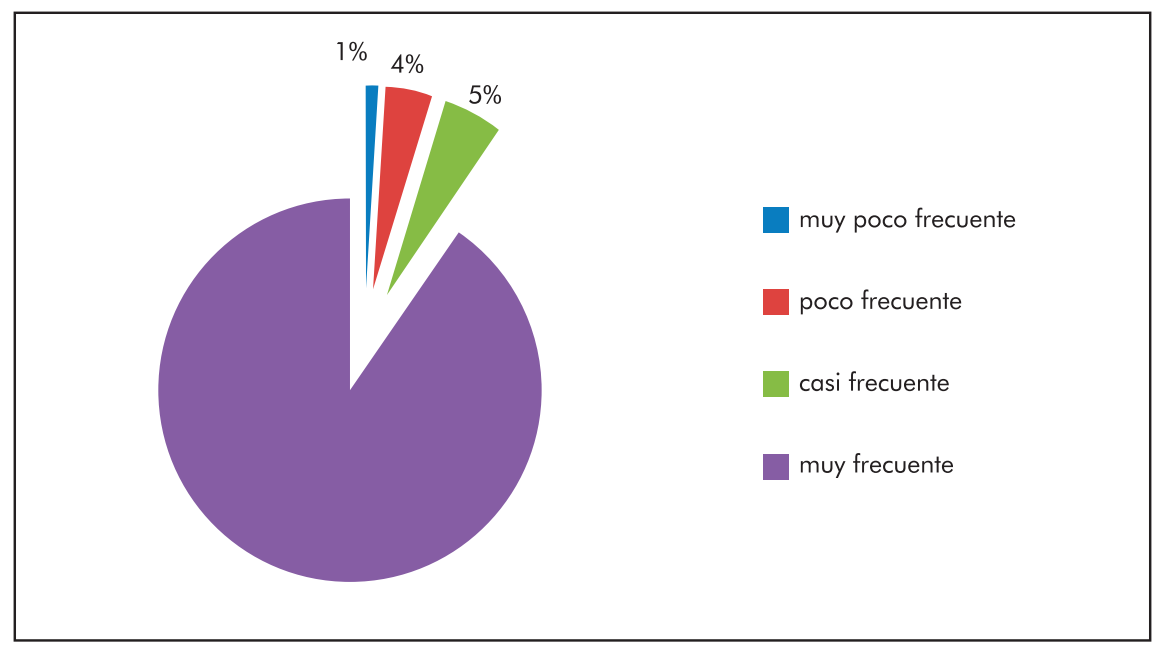

Figura $\mathrm{N}^{\circ}$ 1: Frecuencia de utilización del componente de crecimiento y desarrollo.

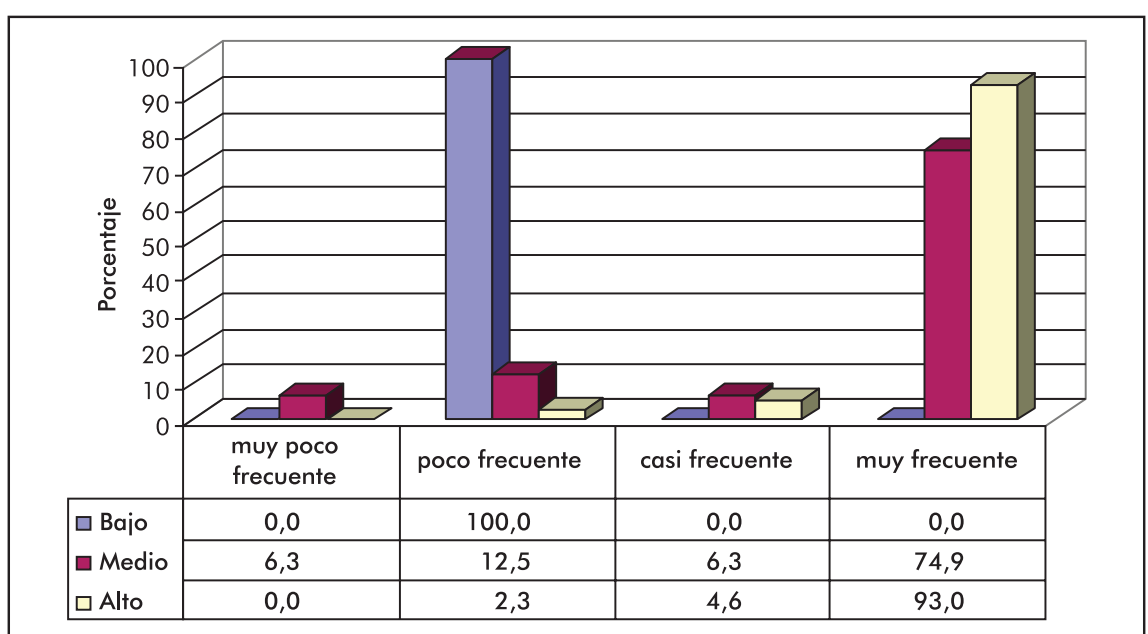

Figura $\mathrm{N}^{\circ} 2$ : Asociación entre el nivel socioeconómico familiar con el acceso y utilización de componente de crecimiento y desarrollo.

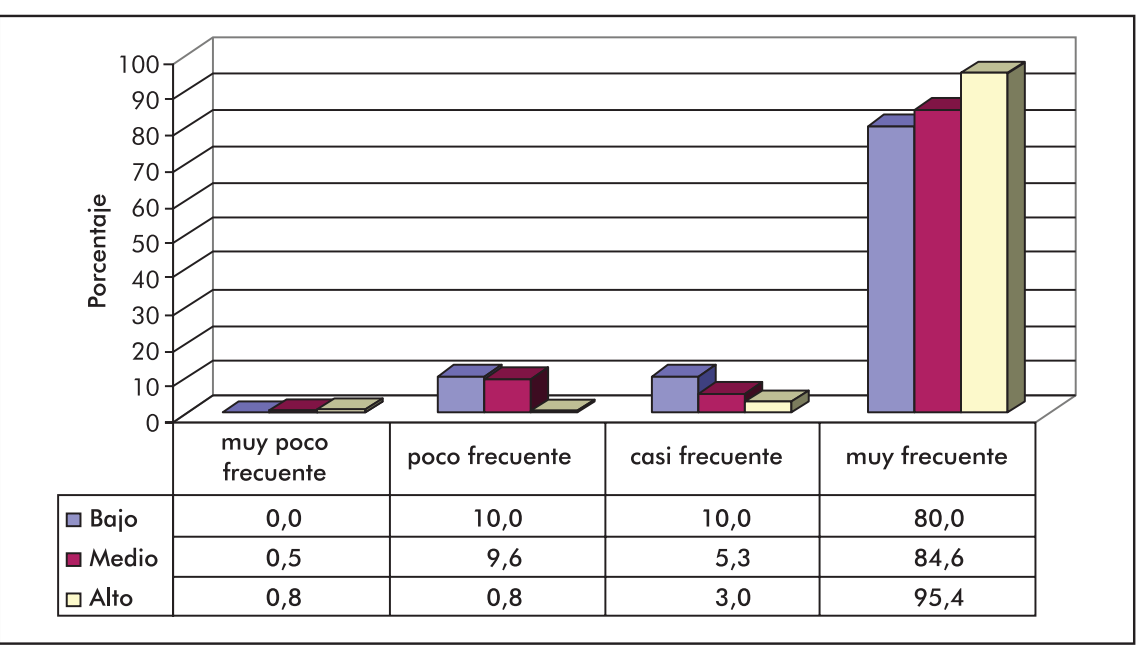

Figura $\mathrm{N}^{\circ}$ 3: Asociación entre el nivel de percepción nutricional y conocimientos sobre el componente CRED con la frecuencia de utilización del componente de crecimiento y desarrollo. 


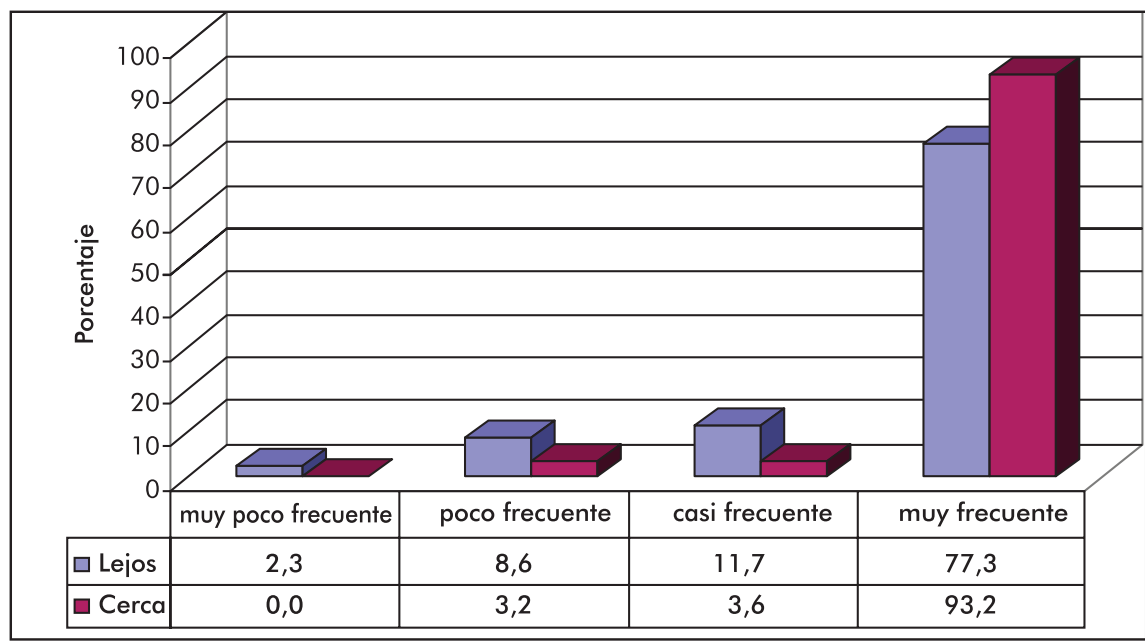

Figura $\mathrm{N}^{\circ}$ 4: Asociación de la percepción de la distancia al establecimiento de salud con el acceso y utilización del componente de crecimiento y desarrollo.

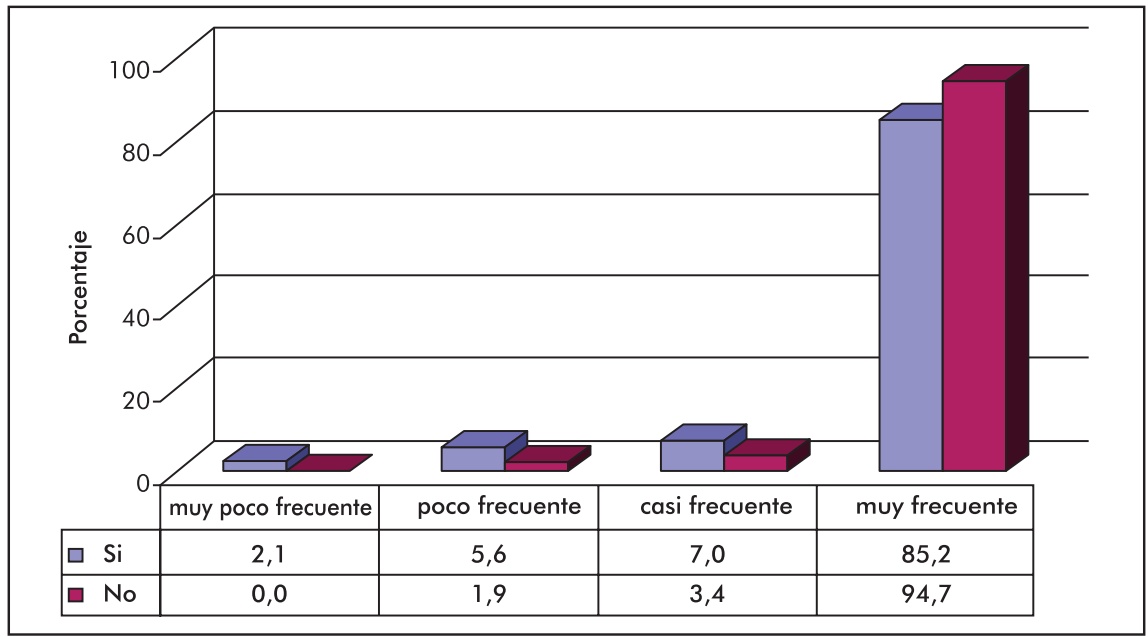

Figura $\mathrm{N}^{\circ} 5$ : Asociación entre los trámites para obtener una cita con el acceso y utilización del componente de crecimiento y desarrollo.

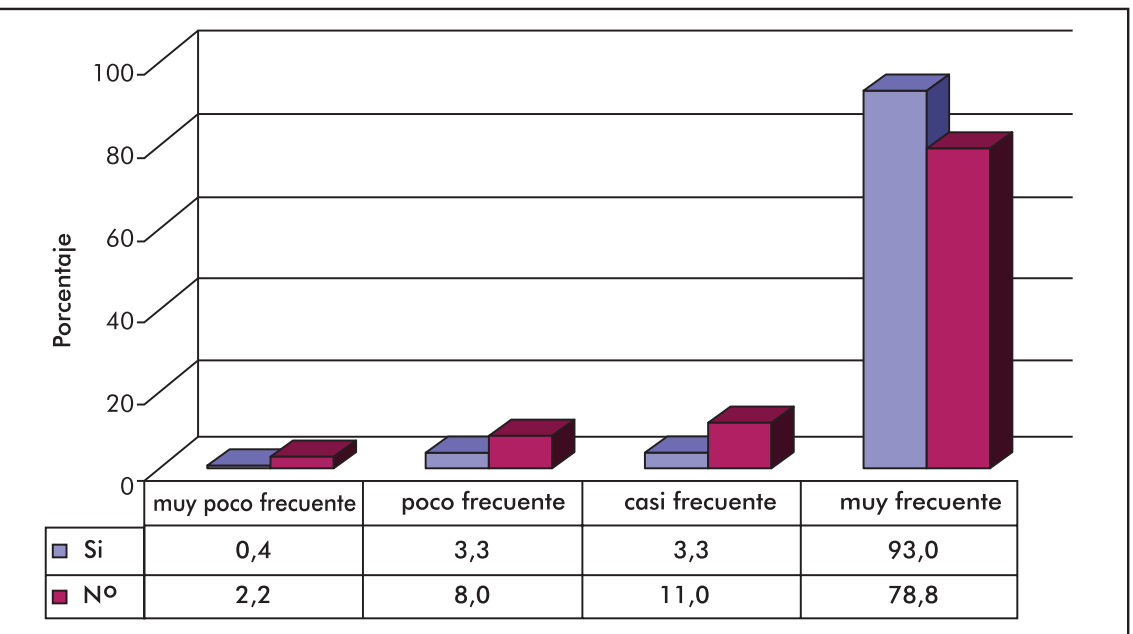

Figura $\mathrm{N}^{\circ}$ 6: Asociación entre la cantidad de profesionales de salud que atiende en el componente con el acceso y utilización del componente CRED. 


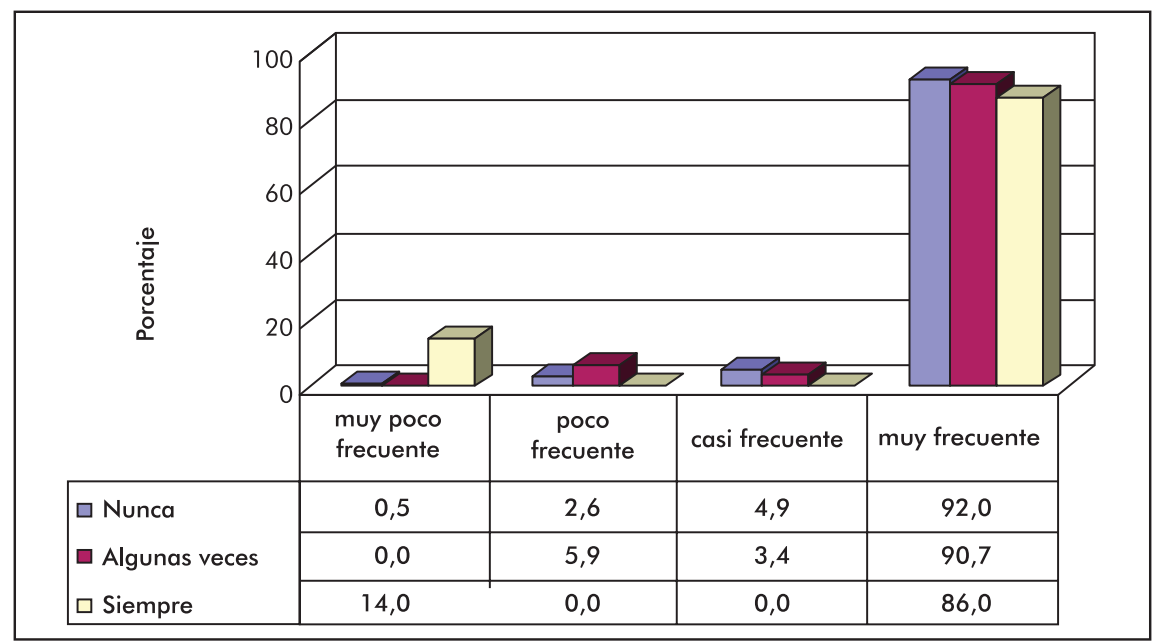

Figura $\mathrm{N}^{\circ}$ 7: Asociación entre la promoción del componente con el acceso y utilización de componente de crecimiento y desarrollo.

En la figura $\mathrm{N}^{\circ} 2$ se puede observar que las madres que acuden muy frecuentemente al componente CRED tienen un nivel socioeconómico alto en un $93 \%$, le sigue el grupo de madres con nivel socioeconómico medio con un $74,9 \%$. Asimismo existe una relación significativa $(p \leq 0,05)$ entre el nivel socioeconómico de las madres y la frecuencia de la utilización del componente CRED, es decir, que a mayor nivel socio económico mayor frecuencia en la utilización del componente CRED.

En la figura $\mathrm{N}^{\circ} 3$ se observa que las madres que acuden muy frecuentemente al componente CRED del CAP II CHILCA, tienen un alto nivel de percepción nutricional de 95,4 \%; y de un medio nivel de percepción nutricional de $84,6 \%$. Así mismo de un bajo nivel de percepción nutricional de un 80 $\%$. Existiendo asociación significativa entre ambas variables $(p \leq 0,05)$.

En la figura $\mathrm{N}^{\circ} 4$ se observa que las madres que acuden muy frecuente al componente CRED tienen una percepción en relación a la distancia al establecimiento de salud de cerca en $93,2 \%$, y de lejos con $77,3 \%$. Existiendo una asociación significativa entre ambas variables con el $95 \%$ de nivel de confianza. Quiere decir que las madres que perciben que su casa está cerca al establecimiento de salud acuden más frecuentemente al componente CRED.

En la figura $\mathrm{N}^{\circ} 5$ se observa que las madres que acuden muy frecuente al componente CRED consideran que los trámites para obtener una cita son muy excesivos en un $85,2 \%$ y los que no consideran que los tramites son excesivos en un 94,7\%. Existiendo una asociación significativa entre ambas variables $(p \leq 0,05)$. Quiere decir que quienes consideran que los trámites no son excesivos acuden con más frecuencia al componente CRED.
En la figura $\mathrm{N}^{\circ} 6$ se observa que las madres que acuden muy frecuente al componente CRED creen que la cantidad de profesionales que atienden a este componente son suficientes en un $93 \%$ y no lo son en un $78,8 \%$. Existiendo una asociación significativa entre ambas variables al $95 \%$ de nivel de confianza. Quiere decir que las madres que perciben que no existe la cantidad de profesionales de salud suficientes acuden menos al componente CRED.

En la figura $N^{\circ} 7$ se observa que las madres que acuden muy frecuentemente al componente CRED del CAP II CHILCA y nunca acceden a la información sobre el componente es de un $92 \%$, algunas veces en un 90,7 $\%$ y siempre en un $80 \%$. Asimismo existe una relación significativa $(p \leq 0,05)$ entre el acceso de información del componente CRED y la utilización del servicio de este componente. Quiere decir que las madres a pesar que no acceden a información detallada sobre el componente acuden muy frecuentemente al servicio.

\section{DISCUSIÓN}

En este estudio realizado a 350 madres con niños menores de 5 años de edad asegurados adscritos pertenecientes al CAP II Chilca ESSALUD de los diferentes distritos del extremo sur de Huancayo, incluido el distrito de Chilca, se encontraron factores limitantes asociados al acceso y utilización del componente de crecimiento y desarrollo de la siguiente manera:

Con respecto al tamaño del grupo familiar en nuestro estudio no está asociado significativamente $(p \geq 0,05)$ a la frecuencia de utilización del componente CRED coincidiendo con el estudio realizado por Mendoza PM, Zegarra RA (11) quienes mencionan que el número de miembros de la familia no está asociado 
con la participación de los padres en el control de crecimiento y desarrollo de sus hijos.

En cuanto al nivel educativo de la madre y la frecuencia de utilización del componente CRED se encontró asociación significativa en ambos $(p<0,05)$, cuando la madre es de un mayor nivel educativo acude con mayor frecuencia al CAP. II CHILCA para el control de crecimiento y desarrollo, se deduce que cuando la persona posee mayor nivel educativo (profesional y secundaria), tiene mejor conocimientos sobre los cuidados a la salud de su hijo y por lo tanto mayor preocupación por la salud, esto explicaría la frecuencia de utilización como muy frecuentemente al componente de CRED, lo que sucede lo contrario con las madres con nivel educativo ninguno o primario; resultados similares muestran los estudios de Mendoza PM, Zegarra R. (11) y Mejía A. (12) quienes muestran como un factor importante al nivel educativo para el acceso y utilización de los servicios de salud.

El ingreso económico familiar mensual, no mostró asociación significativa $(p \geq 0,05)$ con la frecuencia de utilización del componente CRED del CAP II CHILCA; difiere con Rodríguez Acosta (13) en su investigación menciona como una gran barrera al aspecto económico tanto para la población con seguro de salud, como para la población sin él; Vargas Lorenzo I. (14) menciona que el bajo nivel de ingresos de la población es uno de los factores determinantes para el acceso a los servicios de salud del mismo modo otros autores como Mendoza P., Zegarra R. (11), Lama More A. (15) Madueño Dávila M. (16) señalan que la población pobre es la que menos acude a los servicios de salud, siendo esta la más perjudicada.

El nivel socioeconómico que engloba a las tres variables indicadas anteriormente está asociado a la frecuencia de utilización del componente de Crecimiento y Desarrollo $(p \leq 0,05)$, interpretándose que las familias con un nivel socioeconómico alto utilizan con mayor frecuencia el Componente de crecimiento y desarrollo, debido a que estas familias presentan mayores ingresos económicos, mejor nivel educativo, coincidiendo con Mejía A. (12), Rodríguez Acosta, (13) Vargas Lorenzo, (14) estos autores en su tesis mencionan al nivel socioeconómico como un factor determinante en el acceso y utilización de los servicios de salud.

En cuanto al significado real del conocimiento del Componente CRED de las madres con sus hijos que acuden al establecimiento de salud, se encuentra asociado significativamente $(p \leq 0,05)$ a la frecuencia de utilización del componente CRED del CAP II CHILCA, quiere decir que a mayor conocimiento del componente CRED mayor utilización del servicio, este resultado no coincide con el estudio de Jáuregui Cárdenas (17) en el cual hace énfasis en el significado que las madres le atribuyen al Componente de Crecimiento y Desarrollo, menciona que la mayoría de las madres entrevistadas en su estudio le dan un concepto errado, dando la mayor prioridad e importancia a las vacunas, dejando de lado el control de crecimiento y desarrollo. En nuestro estudio el $84,6 \%$ y 95,4 \% presentan conocimientos medio y alto respectivamente sobre el significado del componente CRED.

Con respecto a la variable "percepción del estado nutricional del niño" se halló asociación significativa $(p \leq 0,05)$ con la frecuencia de utilización del componente de crecimiento y desarrollo del CAP II CHILCA, lo que indica que las madres que mejor perciben el estado nutricional de sus niños acuden con mayor frecuencia al componente CRED del establecimiento, los resultados en este estudio coinciden con el de Irwin Rosenstock, donde menciona en su modelo "creencias en salud" que si las personas hacen el problema perceptible "presencia de peligro" adoptan la conducta saludable de acudir al centro asistencial, lo que ocurre lo contrario cuando creen que no existe el problema "no peligro" no adoptan conductas saludables en este caso el no acudir al establecimiento de salud de manera preventiva; de igual manera Mendoza P. (1 1) en su estudio menciona que la percepción de la posibilidad actual de desnutrición está asociado a las visitas frecuentes del CRED. (Odds Ratio 1,79), en nuestro estudio donde se halló que las madres que piensan que sus hijos están desnutridos los llevan con mayor frecuencia al control del crecimiento y desarrollo.

En cuanto a la accesibilidad geográfica se evaluaron dos variables: el tiempo de demora para llegar al establecimiento de salud y la percepción de distancia al establecimiento de salud: En tanto al tiempo para llegar al establecimiento de salud resultó ser estadísticamente significativo $(p \leq 0,05)$ en este estudio; Quiere decir que a menor tiempo de demora para llegar al establecimiento mayor es la frecuencia en la utilización del componente CRED. Coincidiendo con los estudios de Rodríguez Acosta (13) y Sánchez Ruiz (15) realizados en poblaciones dispersas donde el tiempo es un factor altamente asociado al acceso y utilización sobre todo en la zona rural, de otro modo Lama More (15) menciona en su tesis que una de las variables con relación positiva a la utilización de servicios de salud al menor tiempo de llegada. La segunda variable analizada percepción de distancia al establecimiento se encontró asociación significativa $(p \leq 0,05)$ con la frecuencia de utilización del Componente de Crecimiento y Desarrollo, quiere decir que las madres que perciben que su casa está cerca al establecimiento de salud acuden más frecuentemente al componente CRED. Coincide con el estudio de Rodríguez Acosta (13) donde se halló como factor importante la distancia hacia el establecimiento de salud, siendo los más perjudicados quienes perciben 
la lejanía al establecimiento de salud.

Con respecto al tiempo promedio de espera para ser atendidos en el estudio se asocia significativamente $(p \leq 0,05)$ a la frecuencia de utilización. Existiendo una asociación significativa entre ambas variables con un $95 \%$ de nivel de confianza. Lo que quiere decir que a menor tiempo promedio de espera es mayor la frecuencia de utilización del componente CRED. Datos similares encontrados en la investigación de Rodríguez Acosta (13) quien menciona que las personas que no son atendidas rápidamente y tienen que esperar largas horas para ser atendidos son las que utilizan con menor frecuencia los servicios de salud.

En relación a los trámites para obtener una cita para el componente CRED están asociadas significativamente $(p \leq 0,05)$ a la frecuencia de utilización del componente de crecimiento y desarrollo, quiere decir que quienes consideran que los tramites no son excesivos acuden con más frecuencia al componente CRED, coincide con Rodríguez Acosta (13) que a demasiados trámites para la cita las personas dejan de utilizar el servicio de salud.

En relación a la cantidad suficiente de profesionales de salud que atienden en el componente CRED muestra que existe una asociación significativa $(p \leq 0,05)$, ya que las madres que afirman que existe poco personal suficiente en el servicio no acuden al componente CRED este aspecto coincide con Lama More (15) quien menciona en su tesis que el $28,7 \%$ de la población que no acudió al servicio de salud fue por razones estructurales, falta de atención y personal ausente datos similares encontrados en este estudio, del mismo modo Jáuregui Cárdenas (11) en su tesis menciona que una de las razones principales por la cual las madres no acuden al control de crecimiento y desarrollo es la falta de personal, resultados similares a este estudio, del mismo modo Vargas Lorenzo (14) menciona que una de las principales barreras en el acceso a los servicios de salud son las deficiencias estructurales organizativas de parte de la oferta.

Con respecto a la percepción de la calidad de atención al usuario nos muestra una asociación significativa $(p \leq 0,05)$ con respecto a la frecuencia de utilización del componente CRED. Quiere decir que las madres que acuden muy frecuentemente al componente CRED perciben que la atención de este componente es de buena calidad en un 90,7 \% que coincide con el estudio de Sánchez Ruiz (15) quien menciona que la calidad de la asistencia prestada influye sobre la utilización del servicio, resultados similares encontrados en este estudio.

En relación a la información sobre la importancia del Componente CRED resultó ser estadísticamente significativo $(p \leq 0,05)$. Quiere decir que las madres a pesar que no acceden a información detallada (92\%) sobre el componente acuden muy frecuentemente al servicio. Este resultado no coincide con los estudios de Lama More (15) quien menciona a la falta de oferta (promoción) de servicios de salud como un factor determinante para la utilización de servicios de salud.

Algunas conclusiones a las cuales se arribaron son:

1. Los factores limitantes asociados son: el nivel socioeconómico, accesiblidad geográfica, creencias en salud, características de organización interna y la calidad de atención.

2. En relación a las variables socioeconómicas del usuario asociadas con el acceso y utilización del componente CRED en niños de 0 a 5 años en el CAP II Chilca 2014, son significativas $(p \leq 0,05)$ el nivel socioeconómico alto y el nivel de instrucción de la madre.

3. En relación al significado del componente CRED para la madre y la percepción del estado nutricional del niño asociado con el acceso y utilización del Componente crecimiento y Desarrollo en niños de 0 a 5 años en el CAP II CHILCA ESSALUD 2014, los factores limitantes significativos $(p \leq 0,05)$ son: percepción del buen estado nutricional del niño y el conocimiento del significado real del componente.

4. En cuanto a la variable de la accesibilidad geográfica asociado con el acceso y utilización del Componente crecimiento y Desarrollo en niños de 0 a 5 años en el CAP II CHILCA ESSALUD 2014, los factores limitantes significativos $(p \leq 0,05)$ son: la distancia al establecimiento de salud y el tiempo de demora en llegar al establecimiento de salud.

5. En relación a las características de la organización interna del establecimiento de salud asociado con el acceso y utilización del Componente crecimiento y Desarrollo en niños de 0 a 5 años en el CAP II CHILCA ESSALUD 2014, los factores limitantes significativos $(p \leq 0,05)$ son: el tiempo promedio de espera para ser atendido, los trámites para la obtención de una cita y el personal de salud suficiente.

6. En relación a la calidad de atención del usuario y la promoción del componente salud asociado con el acceso y utilización del Componente crecimiento y Desarrollo en niños de 0 a 5 años en el CAP II CHILCA ESSALUD 2014, los factores limitantes significativos $(p \leq 0,05)$ son: la percepción de calidad de atención (buena) y la promoción del componente.

7.- En relación a la cobertura de utilización del componente CRED a en el CAP II CHILCA 
ESSALUD 2014, se encontró que es muy frecuente el $90 \%$, casi frecuente, poco frecuente y muy poco frecuente en $5 \%, 4 \%$ y $1 \%$ respectivamente.

\section{REFERENCIAS BIBLIOGRÁFICAS}

1. Ministerio de Salud. Plan nacional concertado de salud. Lima: MINSA; 2007.

2. Ministerio de Salud. Norma técnica de salud para el control de crecimiento y desarrollo de la niña y niño menor de 5 años. Lima: MINSA; 2010.

3. Organización Mundial de la Salud. Estadísticas sanitarias mundiales, 2010. Ginebra: OMS; 2010. Disponible en: http://www.who.int/whosis/ whostat/ES_WHS10_Full.pdf

4. Fondo de las Naciones Unidas para la Infancia. Mapa de la vulnerabilidad de la desnutrición crónica infantil. Nueva York: UNICEF; 2007.

5. Instituto Nacional de Estadística e Informática. Indicadores de Resultados de los Programas Estratégicos, Primer Semestre ENDES. Lima: INEI; 2011.

6. Instituto Nacional de Estadística e Informática. Indicadores de Resultados de los Programas Estratégicos, Primer Semestre ENDES. Lima: INEI; 2010.

7. Gobierno Regional de Junín. Plan de Desarrollo Regional Concertado Junín: 2008 - 2015. Huancayo: Gob. Regional de Junín; 2008.

8. Dirección Regional de Salud Junín. Plan Estratégico Institucional: Huancayo: DIRESA; 2011.

9. Hernández SR, Fernández CC, Baptista LP. Metodología de la investigación. $5^{\mathrm{a}}$ ed. México D.F.: Mc-Graw Hill; 2010.

10. EsSalud [Internet]. Lima: Seguro Social de Salud del Perú; [Citado el 20 de noviembre de 2014]. Intranet [ 1 pantalla]. Disponible en: http://www. essalud.gob.pe/

11. Mendoza P, Zegarra R, Ríos R, Rivera J. Factores asociados a la participación de la comunidad en campañas de control de crecimiento infantil en "Primavera", Lima - Perú. Rev. peru. Epidemiol. 1993; 6(2): 18-23.

12. Mejía $A F$, Sánchez GA. Determinantes del acceso a servicios de salud en Antioquia. [Tesis de pregrado]. Medellín: Universidad de Antioquia; 2004.

13. Rodríguez S. Barrerás y determinantes del acceso a los servicios de salud Colombia. [Tesis de maestría]. Barcelona: Universidad Autónoma de Barcelona; 2009.

14. Vargas I. Barreras en el acceso a la atención en salud en modelo de competencia gestionada: un estudio de caso en Colombia. [Tesis doctoral]. Bellatona: Universidad Autónoma de Barcelona; 2009.
15. Lama A. Determinantes del acceso a los servicios de salud en el Perú. Lima: Centro de investigaciones INEI; 2003.

16. Madueño M. Perú: Estudio de demanda de servicios de salud. Lima: Socios para la reforma del sector salud, Abt Associates Inc; 2002.

17. Jáuregui JL. El componente crecimiento y desarrollo: significado para las madres de niños de 1 a 4 años del AAHH Nueva Unión, Comas - 2009. [Tesis de pregrado]. Lima: Universidad Nacional Mayor de San Marcos; 2010 